\title{
Michael Heinrich \\ Zum Verhältnis sozialwissenschaftlicher und naturwissenschaftlicher Formen des Wissens
}

Zusammenfassung: Im Hinblick auf einen normativen Wissenschaftsbegriff, der angeblich aus der Praxis der Naturwissenschaften gewonnen wurde, sprechen Wissenschaftstheoretiker aus dem Umkreis von Neopositivismus und Kritischem Rationalismus einem großen Teil der Sozialwissenschaften und insbesondere dem Marxismus jede Wissenschaftlichkeit ab. Wie eine kritische Wissenschaftsgeschichtsschreibung aber zeigte, ist auch die Entwicklung der Naturwissenschaften nicht diesem normativen Ideal gefolgt. Die tatsächliche Entwicklung der Naturwissenschaften läßt sich nicht allein aufgrund einer internen Rationalität erklären, sondern verweist auf ihre sozialgeschichtliche Bedingtheit. Statt daß die angebliche Form naturwissenschaftlichen Wissens einen Maßstab für die Wissenschaftlichkeit der Sozialwissenschaften abgibt, wird deutlich, daß sozialwissenschaftliches Wissen notwendig ist, um die Form naturwissenschaftlichen Wissens adäquat zu begreifen.

\section{Normierung der Sozialwissenschaften durch die Form des naturwissenschaftlichen Wissens?}

Im 19. Jahrhundert erlebten Naturwissenschaften und Technik einen bis dahin einmaligen Aufschwung. Dieser Aufschwung veränderte auch das allgemeine Gefüge der Wissenschaften. Es fand nicht nur eine immer weitergehende Auffächerung und Spezialisierung statt. Vor allem standen sich immer deutlicher zwei getrennte Wissenschaftsbereiche gegenüber: auf der einen Seite Mathematik und experimentelle Naturwissenschaften, auf der anderen Seite Geistes- und Sozialwissenschaften. Der rasche Aufstieg der Naturwissenschaften, ihr offensichtlicher Erfolg bei der Erklärung einer wachsenden Zahl von Naturerscheinungen und der Umsetzung dieser Erkenntnisse in technische Verfügungsmöglichkeiten, machte sie mehr und mehr zum Inbegriff von erfolgreicher Wissenschaft schlechthin. Die Erfolge der Naturwissenschaften wurden zum Maßstab, an dem sich die übrigen Wissenschaften messen lassen mußten. Die Reflexion auf die unterschiedlichen Formen von naturwissenschaftlichem und geisteswissenschaftlichem Wissen war daher stets auch Ausdruck eines Konkurrenzverhältnisses dieser beiden Wissenschaftsbereiche.

Im »Methodenstreit«, der gegen Ende des 19. Jahrhunderts in der deutschen Ökonomie geführt wurde, kamen diese unterschiedlichen Wissenskonzeptionen bereits deutlich zum Ausdruck. Die »theoretisch « orientierte Richtung um Menger faßte Ökonomie als eine deduktive Wissenschaft auf, deren Gesetzesbegriff eine große 
Ähnlichkeit mit dem in den Naturwissenschaften gebräuchlichen hatte. Dagegen zielte die »historische Schule « um Schmoller auf das Ganze der ökonomischen Verhältnisse, von denen angenommen wurde, daß sie sich in ihrer historischen Einzigartigkeit nicht auf einige abstrakte, deduktiv gewonnene Gesetze reduzieren ließen. Der Sieg der »theoretischen « Schule im Methodenstreit, wie auch die Durchsetzung der Grenznutzenlehre und der mathematisch formulierten allgemeinen Gleichgewichtstheorie zum herrschenden ökonomischen Paradigma, paßte in einen wissenschaftlichen Zeitgeist, der vom Fortschritt der Naturwissenschaften geprägt war.

Im Neukantianismus wurde das Thema dann grundsätzlicher aufgerollt. Bereits Windelband (1894) hatte den auf allgemeine Gesetze ausgerichteten (nomothetischen «) Naturwissenschaften, die das Besondere, Einmalige, Individuelle untersuchenden (»idiographischen«) Kulturwissenschaften gegenübergestellt. Rickert (1902) versuchte im Anschluß daran auf transzendentalphilosophischer Grundlage die Geisteswissenschaften selbständig zu begründen. Während sich die Erscheinungen durch die Kategorien des Verstandes unter allgemeinen Gesetzen zu »Natur«konstituieren würden, sei es ihre individuelle Beziehung auf ein System von Werten, die sie zu »Kultur « macht. Die Sozialwissenschaften wurden von Max Weber (1922) schließlich als Kombination des »erklärenden« Verfahrens der Naturwissenschaften und des »verstehenden « der Geisteswissenschaften aufgefaßt. Es sollen zwar allgemeine Gesetze sozialen Handelns aufgestellt werden, Weber geht aber davon aus, daß soziale Prozesse im Unterschied zu Naturprozesssen durch den Nachvollzug ihrer Motivationen auch verständlich sind.

Mit dem erkenntnistheoretischen Dualismus von Naturwissenschaften einerseits und Geistes- und Kulturwissenschaften andererseits war ein prekäres Gleichgewicht hergestellt worden. Radikal in Frage gestellt wurde dieses Gleichgewicht durch den Positivismus des Wiener Kreises um Rudolf Carnap. Nur Sätze, die empirische Beobachtungen ausdrücken oder sich aus ihnen ableiten lassen, wurden überhaupt als »sinnvoll « anerkannt (Carnap 1928). Alles andere seien sinnlose Sätze, die die Grundlage von »Scheinproblemen « bildeten, mit denen sich die bisherige Philosophie vorwiegend beschäftigt habe. In der Entlarvung solcher Scheinprobleme wurde die einzige verbleibende Aufgabe der Philosophie gesehen. »Wovon man nicht sprechen kann, darüber muß man schweigen«, lautete schon die bekannte Folgerung des frühen Wittgenstein (1921).

Die Naturwissenschaften sollten jetzt das Modell für Wissenschaft schlechthin abgeben. Das von Carnap und Neurath vertretene Ziel war eine »Einheitswissenschaft«, deren Grundlage die »Dingsprache« sein sollte. Letzten Endes sollten auch alle $\mathrm{Hu}-$ man- und Gesellschaftswissenschaften ihre Grundlage in der Physik finden. Der Positivismus des Wiener Kreises richtete sich zwar in erster Linie gegen die als »metaphysisch « aufgefaßten Probleme der Philosophie, allerdings galt Theoriebildung, die dem dabei entstandenen normativen Wissenschaftsbegriff nicht entsprach, als metaphysisch.

Das erkenntnistheoretische Programm des Wiener Kreises wurde von Popper (1935) einer eingehenden Kritik unterzogen. Es stellte vor allem klar, daß Sätze nur durch 
Sätze, aber nicht durch Beobachtungen bewiesen werden können. Davon abgesehen ist es auch nicht möglich ein Gesetz, das eine Allaussage umfaßt, zu beweisen, da nie alle Fälle überprüft werden können. Es ist nur möglich, ein solches Gesetz durch ein Gegenbeispiel zu widerlegen. Statt Verifizierbarkeit machte Popper Falsifizierbarkeit zum Kriterium für eine wissenschaftliche Aussage, zur »Demarkationslinie« zwischen Wissenschaft und Nicht-Wissenschaft, wie er selbst es nannte. Popper unterschied nicht sinnlose von sinnvollen Sätzen, sondern falsifizierbare von nicht-falsifizierbaren Sätzen. Von einer wissenschaftlichen Theorie verlangte er nicht, daß sie ausschließlich falsifizierbare Sätze enthalten müsse, aber er verlangte, daß wenigstens einige Aussagen aus ihr abgeleitet werden können, die falsifizierbar sind. Entgegen dem positivistischen Programm war damit ein viel weiterer Bereich der Theoriebildung zugelassen. Vor allem änderte sich aber auch die Vorstellung vom wissenschaftlichen Fortschritt. Er wurde nicht mehr als Entwicklung von immer umfassenderen und durch Induktion aus Beobachtungen immer besser verifizierten Theorien aufgefaßt; die Hauptaufgabe der Wissenschaft bestand jetzt darin, nach Falsifikationen für die vorhandenen Theorien zu suchen, und wenn eine Theorie falsifiziert war, sie durch eine andere zu ersetzen.

In seiner »Logik der Forschung « beanspruchte Popper zwar, die Verfahrensweise der Naturwissenschaften zu beschreiben, er ging aber sehr schnell von einer deskriptiven zu einer normativen Argumentation über. Die normativen Vorstellungen von Wissenschaft, die Popper entwickelte und die auch heute in popularisierter Form das methodische Selbstverständnis vieler Wissenschaftler prägen, beziehen ihre Überzeugungskraft aber gerade daraus, daß sie anscheinend die Verfahrensweise der erfolgreichen Naturwissenschaften widerspiegeln. Die internen Probleme des Popperschen Ansatzes sind in diesen populären Auffassungen allerdings verlorengegangen. Nur zwei dieser Probleme sollen hier erwähnt werden. (A) Es ist stets möglich, eine »falsifizierte Theorie durch eine »konventionalistische Wendung «, d.h. durch die Hinzufügung eines weiteren Theorems zu retten. Hier blieb Popper nur der Appell an die Wissenschaftler, auf solche Wendungen zu verzichten. Ein großer Teil der alltäglichen Forschungspraxis spielt sich aber gerade in dieser konventionalistischen Weise ab. (B) Die »Basissätze«, die empirische Ergebnisse ausdrücken sollen und zur Falsifikation von Theorien dienen, können nur durch Konvention statuiert werden. Jede Beobachtungsaussage kann noch weiter zurückverfolgt werden und ist darüber hinaus selbst theorieabhängig und damit falsifizierbar, womit dann auch die aufgrund dieses Basissatzes erfolgte Falsifikation einer anderen Theorie hinfällig wäre. Wird eine Theorie aufgrund eines Basissatzes falsifiziert, so muß dieser Basissatz vorher per Konvention als wahr akzeptiert worden sein. Kriterien für diesen erlaubten Konventionalismus kann Popper aber nicht angeben.

Poppers »Kritischer Rationalismus « entwickelte sich nach dem 2. Weltkrieg in Westeuropa und den USA zum dominierenden wissenschaftstheoretischen Paradigma. Wer Anspruch auf Wissenschaftlichkeit erhob, mußte sich gegenüber dem Popperschen Wissenschaftsbegriff legitimieren. So versuchte eine empirische Sozialforschung durch verstärkten Rückgriff auf quantitative Methoden falsifizierbare Resul- 
tate zu liefern und dem Verdikt der Unwissenschaftlichkeit zu entgehen. In den nicht nur wissenschaftstheoretischen sondern auch wissenschaftspolitischen Auseinandersetzungen wurde der Poppersche Wissenschaftsbegriff aber vor allem als ein Mittel zur Diskreditierung des Marxismus benutzt. Der immer von neuem wiederholte Vorwurf lautete »Immunisierung «: entweder wären die zentralen Aussagen von vornherein so unklar formuliert, daß eine Falsifizierung durch empirische Ergebnisse gar nicht möglich sei, oder es würde einer Falsifizierung durch immer neue Hilfshypothesen aus dem Wege gegangen.

In der Bundesrepublik fanden diese Auseinandersetzungen im sogenannten $\gg$ Positivismusstreit der Soziologie« einen Höhepunkt. Als Hauptkontrahenten standen sich dabei Popper und Albert auf der einen Seite sowie Adorno und Habermas auf der anderen Seite gegenüber. Popper (1961) beanspruchte, daß dieselbe $\gg$ kritische Methode« von Hypothesenbildung und Widerlegungsversuchen in Natur- und Sozialwissenschaften gleichermaßen Geltung habe, daß sich also auch die Sozialwissenschaften dem Wissenschaftsideal des Kritischen Rationalismus unterwerfen müßten. Die Reaktion von Adorno und Habermas war eher defensiv. So betonte Adorno (1961) die Besonderheit des Sozialen, eine Besonderheit, die auch besondere Verfahrensweisen notwendig machen würde. Da die Gesellschaft selbst widerspruchsvoll, rational und irrational zugleich sei, würde sie sich dem von Popper angestrebten Erklärungsmuster entziehen, die Methode könne nicht unabhängig vom Gegenstand gewählt werden. Die Soziologie dürfe man nicht mit naturwissenschaftlichen Methoden vermengen. Auf einer ähnlichen Linie argumentierte auch Habermas (1963). Sein zentrales Argument bestand ebenfalls darin, daß es eben nicht möglich sei, daß »die Wissenschaft in Ansehung der von Menschen hervorgebrachten Welt ebenso indifferent verfahren darf, wie es in den exakten Naturwissenschaften mit Erfolg geschieht« (Habermas 1963, S. 157). Adorno und Habermas akzeptieren also die Gültigkeit des Popperschen Wissenschaftsbegriffs für die Naturwissenschaften, lediglich für die Sozialwissenschaften lehnten sie ihn aufgrund der Besonderheit des Gegenstandes ab. Zwar geht Habermas noch einen Schritt weiter, indem er sich gegen die Trennung von »Tatsachen«, deren Vorliegen rational zu überprüfen ist, und normativen »Entscheidungen «, die rational nicht mehr zu diskutieren seien, wendet. In dieser Trennung erblickt er einen »halbierten Rationalismus «, der nicht nur ausblenden würde, daß auch normative Entscheidungen rationaler Diskussion zugänglich seien, sondern vor allem, daß auch der wissenschaftliche Forschungsprozeß Teil eines sozialen Prozesses sei und daß erkenntnisleitende Interessen in den Forschungsprozeß eingehen würden. Entgegen dem verbreiteten Gebrauch des Begriffs der »erkenntnisleitenden Interessen« meint Habermas damit aber nicht die äußere Motivation eines Forschers, sondern eine die Wissenschaft intern strukturierende Ausrichtung, die schon existiert, bevor der Forscher an sie herantritt. Als erkenntnisleitendes Interesse der Naturwissenschaft betrachtete Habermas die Beherrschbarkeit und Verfügbarkeit von Naturprozessen (Habermas 1965) und diese Beherrschbarkeit könne für die gesellschaftlichen Prozesse, mit denen es die Sozialwissenschaft zu tun hat, nicht in gleicher Weise unterstellt werden (Habermas 1963). Die Berücksichtigung dieser erkenntnisleiten- 
den Interessen stellt sich aber eher als Ergänzung denn als Kritik an der Wissenschaftstheorie des »Kritischen Rationalismus « dar, denn dieser erscheint als durchaus adäquate Beschreibung der von dem Interesse an technischer Verfügung gesteuerten naturwissenschaftlichen Forschungsprozesse.

\section{Das neopositivistisches Wissenschaftsideal und die historische Entwicklung der Naturwissenschaften}

Das Wissenschaftsideal des $\gg$ Kritischen Rationalismus « bezieht seine Legitimation aus den Erfolgen der Naturwissenschaften, denn anscheinend formuliert dieses Ideal nur deren Methoden. Daß der Poppersche »Falsifikationismus « die Vorgehensweise der Naturwissenschaftler adäquat wiedergibt, wird auch von vielen Sozialwissenschaftlern nicht grundsätzlich in Frage gestellt, insbesondere dann nicht, wenn sie selbst sich nicht mit Wissenschaftstheorie beschäftigt haben. Allerdings zeigte insbesondere in den letzten 30 Jahren eine kritische Wissenschaftsgeschichtsschreibung, daß Forschungspraxis und Entwicklung der Naturwissenschaften keineswegs den methodischen Imperativen des »Kritischen Rationalismus « folgen, sondern daß der Poppersche »Falsifikationismus « durch die Wissenschaftsgeschichte selbst »falsifiziert« wird.

Zu Anfang des Jahrhunderts hatte Duhem mit der Vorstellung, die moderne Physik habe schlagartig mit Galilei und Newton begonnen, aufgeräumt und ihre mittelalterlichen Wurzeln bloßgelegt. Entgegen der von Duhem unterstellten Kontinuität unterschied bereits Koyré (1939) drei verschiedene Typen der Physik: die aristotelische Physik der Antike, die Impetusphysik des Mittelalters und die Galileische Physik der Neuzeit. Zwar versuchte Anneliese Maier (1949) noch einen erfahrungswissenschaftlich begründeten Übergang von der Impetusphysik zur Galileischen Physik nachzuweisen, ihre Argumente waren aber nicht sehr überzeugend (vergl. z.B. Wolff 1978, S.46f.). Unter den jüngeren Wissenschaftshistorikern herrschte die Meinung vor, daß es nicht möglich ist, die Übergänge zwischen diesen Typen physikalischer Theoriebildung empiristisch aufgrund von neuen Beobachtungsergebnissen zu erklären (vergl. z.B. Kuhn 1962, Butterfield 1966, Feyerabend 1975, Wolff 1978). Wie wenig die wirkliche Entwicklung der Naturwissenschaften mit den Vorstellungen des Popperschen »Falsifikationismus « übereinstimmt, soll hier an einem Beispiel, der »Kopernikanischen Wende«, illustriert werden.

In vielen mehr oder weniger populären Darstellungen der Entstehung und Durchsetzung des kopernikanischen Systems wird etwa folgendes Bild vermittelt: mit zunehmender Genauigkeit der Beobachtung der Planetenbewegung mußten in das ursprüngliche, geozentrische Modell des Ptolemäus immer mehr Epizykel eingeführt werden, damit es mit den Beobachtungstatsachen übereinstimmte. Dabei wurde immer deutlicher, daß es sich um künstliche ad-hoc Konstruktionen handelte. Das heliozentrische Modell von Kopernikus dagegen war einfacher und stimmte mit den Beobachtungsdaten genauer überein. Keplers Ersetzung der kreisförmigen Planeten- 
bahnen durch Ellipsen verbesserte das Modell nochmals. Und schließlich konstruierte Galilei ein Teleskop, wodurch sich nicht nur die Beobachtungsgenauigkeit erhöhte; durch Galileis Entdeckung der Venusphasen wurde das ptolemäische Modell endgültig falsifiziert. Letzteres ist auch die Meinung von Popper (1969, S. 246). Bei der kopernikanischen Wende scheint es sich also um ein Musterbeispiel für die Falsifikation einer Theorie durch genauere Beobachtungsdaten und die Entwicklung einer neuen Theorie, die mit diesen Daten übereinstimmt, zu handeln.

Nur stimmt dieses Bild nicht sehr gut mit dem überein, was die Wissenschaftsgeschichtsschreibung inzwischen an Material erbracht hat. Das kopernikanische Modell war weder einfacher noch genauer als die gängigen ptolemäischen Modelle. Im ersten Buch von $\gg$ De revolutionibus « gibt Kopernikus eine populäre qualitative Beschreibung seines Systems und stellt deren Einfachheit heraus. Die in den folgenden Büchern entwickelte quantitative Version, die zur Berechnung der Planetenörter notwendig ist, enthält aber ähnlich viele Epizykel wie die gebräuchlichen ptolemäischen Systeme. Überdies gab es auch keine neuen Beobachtungsergebnisse, die die Notwendigkeit eines neuen Modells unmittelbar gezeigt hätten (vergl. dazu Lakatos 1976, S. 183-189)

Kopernikus war durch andere Faktoren motiviert als durch eine $»$ Falsifikation $«$ des heliozentrischen Weltbildes. Er wollte die Planetenbewegungen vor allem wieder entsprechend dem platonischen Ideal durch gleichförmige Kreisbewegungen beschreiben. ${ }^{1}$ Die Sonne in den Mittelpunkt der Welt zu rücken, schien Kopernikus wohl auch deshalb weniger unnatürlich als vielen seiner Zeitgenossen, da er von der spätplatonischen Sonnenmystik beeinflußt war (Dijsterhuis 1950, S. 325 f., Kuhn 1957). Bei Kepler war die Motivation durch platonisch-phythagoräische Zahlenharmonien noch viel stärker als bei Kopernikus. Auch die Sonnenverehrung spielte bei ihm eine grössere Rolle: sie motivierte seine Auffassung, die Sonne sei nicht nur die Licht-sondern zugleich die Kraftquelle der Welt. Er will daher nicht nur eine Kinematik, sondern eine Dynamik der Planetenbewegung liefern. Heute wird Keplers Werk in einen wissenschaftlichen und einen zeitbedingten »abergläubischen« Teil zerlegt. Für Kepler galt diese Trennung aber nicht. Gerade der Teil seines Werkes, der heute als wissenschaftlich anerkannt ist, wäre ohne diesen mystischen Teil nicht zustandegekommen.

Die kritisch-rationalistische Wissenschaftstheorie antwortet auf solche Feststellungen mit ihrer Unterscheidung zwischen dem »context of discovery « und dem »context of justification« eines wissenschaftlichen Ergebnisses. Der Kontext einer Entdekkung mag durch individuelle Zufälligkeiten und Irrationalitäten geprägt sein, aber gleichgültig wie die Theorie zustande gekommen ist, sie muß sich dem Experiment als unabhängigem Richter stellen. Und hier soll eine unbestechliche und von jeder Irrationalität freie Instanz existieren, die darüber entscheidet, ob die Theorie aufrechterhalten werden kann, oder ob sie verworfen werden muß. Geht es um die kopernikanische Wende, kommt an dieser Stelle Galilei mit seinem Teleskop ins Spiel. Das Teleskop soll die entscheidende Verbesserung der Beobachtungsdaten gebracht haben, was das Ende der ptolemäischen Theorie bedeutete. Die scholastischen Wissenschaftler, die die Fernrohrbeobachtungen mit der Autorität des Aristoteles bestreiten 
wollen, wirken dagegen lächerlich, was Brecht in seinem »Leben des Galilei« dadurch zum Ausdruck bringt, daß sie sich einfach weigern, durch das Fernrohr zu sehen. Was so lächerlich wirkt, hat aber durchaus einen rationellen Kern. Mit dem Fernrohr wird der unmittelbaren menschlichen Wahrnehmung ein Instrument vorgeschaltet. Es wird nur das wahrgenommen, was das Instrument liefert. Um daraus auf die »Wirklichkeit « zu schließen, ist eine Theorie über die Funktionsweise dieses Instruments erforderlich. Diese war zu Galileis Zeiten aber nur unzureichend entwikkelt (vergl. dazu Feyerabend 1975, S. 128-183). Daß die Forderung nach einer Theorie des Fernrohrs keine Spitzfindigkeit war, zeigten die optischen Täuschungen, die das Gerät bei Beobachtungen auf der Erde hervorrief. Während diese Täuschungen auf der Erde allerdings durch Beobachtungen mit den eigenen Augen als solche erkannt werden konnten, war diese Möglichkeit bei neuentdeckten Himmelserscheinungen verstellt. Es gab also rationale Gründe, Himmelsbeobachtungen durch das Fernrohr zumindest mißtrauisch gegenüber zu stehen.

Was schließlich Galilei selbst (und heute Popper) als besonders schlagendes Argument für das heliozentrische System empfand, die Entdeckung der Venusphasen, konnte auch durch das Modell von Tycho Brahe erklärt werden: in diesem System drehen sich zwar die Planeten um die Sonne, die Sonne dreht sich aber mitsamt den Planeten um die Erde.

Und schließlich gab es noch einen auch schon in der Antike diskutierten Sachverhalt, der gegen das heliozentrische Weltbild sprach: die fehlende Fixsternparallaxe. Bewegt sich die Erde um die Sonne, so müßte sich diese Bewegung in einer scheinbaren Bewegung der einzelnen Fixsterne niederschlagen. Daß diese Parallaxe nicht wahrgenommen wird, bedeutet entweder, daß die Erde in Relation zu den Fixsternen ihre Position nicht ändert oder daß die Fixsterne so weit entfernt sind, daß dieses Parallaxe nicht wahrnehmbar ist. Dann muß aber die Entfernung zwischen der Erde und der Sonne, im Vergleich zur Entfernung Erde-Fixstern, extrem klein sein. Diese Folgerung muß man aus dem kopernikanischen System ziehen. Daß der Himmel, der bisher immer als eine Einheit gesehen wurde, eine solche bizarre Struktur aufweisen sollte, sprach bei dem damaligen Stand der Wissenschaft eher gegen das kopernikanische Modell. Erst 1838 wurde die Fixsternparallaxe von Bessel entdeckt. Erhebt man diese Entdeckung im Nachhinein zum experimentum crucis zwischen geozentrischem und heliozentrischem Modell, so hätte sich das heliozentrische Modell aber schon fast 200 Jahre vor der Falsifizierung des geozentrischen Modells durchgesetzt. Daß sich das kopernikanische Weltbild so schnell durchsetzte, läßt sich mit dem Popperschen Falsifikationismus nicht erklären.

Kopernikus selbst hob zwar die Einfachheit seines Systems gegenüber dem Ptolemäischen hervor, dies gilt aber nur für die qualitative Beschreibung seines Modells, nicht für die quantitative Darstellung. Auch die Tatsache, daß eine Reihe von bekannten Beobachtungstatsachen, wie etwa die Existenz von Haltepunkten und rückläufigen Planetenbewegungen oder die beschränkte Elongation der inneren Planeten gegenüber der Sonne im Rahmen des heliozentrischen Modells eine ganz natürliche Erklärung finden, machte Kopernikus geltend. ${ }^{2}$ Diesen Vorteilen stehen aber auch gewichtige 
Nachteile gegenüber: die nicht beobachtete Fixsternparallaxe wurde bereits erwähnt und allgemein gilt, daß die kopernikanische Kosmologie nicht mit der herrschenden (aristotelischen) Physik zusammenpaßte, nach der die Bewegung der Erde physikalische Effekte hervorbringen müßte, die nicht beobachtet wurden. Die von Kopernikus erreichten Fortschritte hatten ihren Preis. Auch Ptolemäus hatte im Almaget die Möglichkeit der Erdbewegung diskutiert, sie aber verworfen, weil ihm dieser Preis zu hoch war. Die Abwägung zwischen den Vor- und Nachteilen des kopernikanischen Systems läßt sich nicht mit wissenschaftsinternen Rationalitätsmaßstäben allein entscheiden, denn das, was aufgegeben wird, und das, was gewonnen wird, ist nicht ohne weiteres kommensurabel. Daher scheint es plausibel, daß in eine solche Abwägung auch »externe« Faktoren eingehen. So mag für die Durchsetzung des kopernikanischen Systems in Kreisen fortschrittlich orientierter Gelehrter und Bürger auch eine Rolle gespielt haben, daß es in einer gesellschaftlichen und intellektuellen Umbruchphase entstand und daß es sich in der Auseinandersetzung mit der alten von kirchlichen Autoritäten repräsentierten Ordnung instrumentalisieren lie $3 .^{3}$

Anhand der kopernikanischen Wende läßt sich anschaulich illustrieren, daß die Dynamik der Wissenschaftsentwicklung nicht in der Weise stattfindet, wie sich sich aufgrund der methodologischen Regeln des kritischen Rationalismus abspielen soll. Dabei handelt es sich aber keineswegs um einen Einzelfall. Kuhn (1962) entwarf aufgrund solcher Beispiele ein Bild der Wissenschaftsgeschichte, das den neopositivistischen Vorstellungen radikal zuwiderläuft. Wissenschaft wird für ihn nicht durch Theorien, sondern durch »Paradigmen « konstituiert, Vorstellungen vom Wesen wissenschaftlicher Erkenntnisse, von der gebotenen wissenschaftlichen Vorgehensweise etc. Ein solches Paradigma wird nicht als Sammlung abstrakter Aussagen formuliert, sondern im Rahmen der wissenschaftlichen Ausbildung anhand beispielhafter Problemlösungen gelernt und verinnerlicht. An diesem Paradigma orientieren sich die Wissenschaftler dann in ihrem eigenen Vorgehen, ihrer Begriffsbildung und ihrer Problemdefinition. In der Entwicklung der Wissenschaften unterscheidet Kuhn die $\gg$ normale Wissenschaft $\ll$ von den »wissenschaftlichen Revolutionen $\ll$. Die normale Wissenschaft ist dadurch charakterisiert, daß ein allgemein akzeptiertes Paradigma vorliegt und sich die wissenschaftliche Forschung ihm Rahmen dieses Paradigmas bewegt. Zwar kommt es immer vor, daß Fragen auftauchen, die sich nicht ohne weiteres im Rahmen dieses Paradigmas lösen lassen, sie werden aber als »noch «nicht gelöste Probleme beiseite gestellt. Nehmen solche Anomalien überhand, werden sie als besonders schwerwiegend angesehen und schwindet die Hoffnung auf ihre Lösung, so kann es zu einer Krise des Paradigmas kommen, die in einer wissenschaftlichen Revolution mündet: dem Kampf des alten mit einem neuen Paradigma. Das neue Paradigma bietet Lösungen für Probleme an, die im Rahmen des alten Paradigmas nicht oder nur sehr schwer zu handhaben waren, allerdings ist es auch mit einem Verzicht auf vorhandene Problemlösungen erkauft. Kuhns provozierende These besteht nun darin, daß zwischen diesen beiden Paradigmen nicht mit wissenschaftsinternen Rationalitätsmaßstäben entschieden werden kann, da solche Maßstäbe nur innerhalb eines Paradigmas zu definieren sind. Der Übergang zu einem neuen Paradigma ist daher 
eher als eine Art von $»$ Bekehrung « zu begreifen, deren Ursachen in der Soziologie der »scientific community « zu suchen ist.

Insbesondere von Lakatos wurde Kuhn Irrationalismus und Relativismus vorgeworfen. In seiner eigenen Konzeption versuchte Lakatos (1968), die gewonnenen wissenschaftsgeschichtlichen Einsichten zu berücksichtigen und trotzdem an allgemeinen Rationalitätskriterien festzuhalten. Auch er betrachtet ähnlich wie Kuhn nicht einzelne Theorien, sondern »Forschungsprogramme « als die grundlegenden Einheiten der Wissenschaftsentwicklung. Ein Forschungsprogramm besteht aus einem harten Kern von Theorien, einer positiven Heuristik, die es erlaubt, neue Probleme zu definieren und einem Schutzgürtel von Hilfshypothesen. Diese Hilfshypothesen können nun immer so angepaßt werden, daß der harte Kern durch Experimente nicht widerlegt wird und das Forschungsprogramm durch »Immunisierung « eine Atempause erhält. Entscheidend für ein Forschungsprogramm sei nicht die Suche nach falsifizierenden Experimenten, sondern die Heuristik, die es erlaubt, Problemstellungen zu formulieren und diese Probleme auch theoretisch zu lösen. Werden die theoretischen Lösungen empirisch bestätigt, so ist das Programm erfolgreich. Versagt das Programm in einzelnen Fällen, so werden diese Fälle nicht als Falsifikation, sondern als Anomalien aufgefaßt und zur Seite gestellt. Ein Forschungsprogramm, das weiterhin erfolgreiche Problemlösungen produziert, kann auch mit einer ganzen Reihe von Anomalien leben. Erst wenn die Problemlösungskapazität abnimmt oder stagniert, ist das Überleben des Forschungsprogramms gefährdet. Werden schließlich keine neuen Tatsachen vorhergesagt, sondern nur noch Problemlösungen eines konkurrierenden Programms adaptiert, spricht Lakatos von einem »degenerierenden« Forschungsprogramm. Zwar muß auch Lakatos zugeben, daß Forschungsprogramme niemals widerlegt werden können, allerdings glaubt er, im Gegensatz zu Kuhn, mit rationalen Mitteln zwischen progredierenden und degenerierenden Forschungsprogrammen unterscheiden zu können und damit eine $»$ rationale Rekonstruktion « der Wissenschaftsgeschichte zu liefern (Lakatos 1970). Bei dieser rationalen Rekonstruktion taucht die wirkliche Geschichte aber nur noch in den Fußnoten auf, wie Lakatos selbstironisch zugeben mußte.

Bei aller Differenz im Detail zeigen die Arbeiten von Kuhn und Lakatos, daß sich die normale Forschungspraxis in den Naturwissenschaften nicht mit dem Popperschen Falsifikationismus beschreiben läßt. ${ }^{4}$ Ein von der Theorie nicht oder falsch vorhergesagtes Ergebnis kann nicht nur bedeuten, daß die zugrundlegende Theorie falsch ist, sie kann auch auf einem Fehler in der Versuchsanordnung oder aber auf einem Interpretationsfehler der Theorie beruhen. Werden die Ergebnisse bei erneuten Versuchen reproduziert, so wird normalerweise auf einen Interpretationsfehler geschlossen oder die Theorie so abgeändert, daß sie zu den Versuchsergebnissen paßt. Also genau das, was vom Kritischen Rationalismus immer wieder als »Immunisierung « in den Sozialwissenschaften bekämpft wird, ist in den Naturwissenschaften eine ganz normale Praxis. Läßt sich das Meßergebnis nicht durch eine Uminterpretation beseitigen, so bleibt es als »Anomalie « bestehen, als etwas, was »noch «nicht erklärt werden kann. Wegen einer Anomalie wird aber keine ansonsten erfolgreiche Theorie aufgegeben. Daß 
Theorien durch entscheidende Experimente zu Fall gebracht worden seien, ist meistens eine nachträgliche Projektion. ${ }^{5}$

Wenn aber in wissenschaftlichen Revolutionen Paradigmen wirklich ausgewechselt werden so ist auch dies keine Folge falsifizierender Experimente. Hier ist auch Lakatos' Versuch einer »rationalen Rekonstruktion « unbefriedigend. Was er als wissenschaftlichen Fortschritt konstatiert, geschieht aus einer späteren Perspektive, indem er einen späteren Stand der Forschung zugrunde legt. In den Zeiten eines wissenschaftlichen Umbruchs muß zwischen verschiedenen Vor- und Nachteilen des neuen Paradigmas (oder Forschungsprogramms) abgewogen werden und da sind die Verhältnisse in der Regel nicht so eindeutig. Und Kriterien wie »Harmonie « oder »grössere Geschlossenheit«, die von Naturwissenschaftlern zuweilen als Begründung für die Attraktivität eines neuen theoretischen Ansatzes angeführt werden, sind nicht objektivierbar. In solchen Werturteilen, die für die Akzeptanz einer neuen Theorie entscheidend sein können, gehen auch wissenschaftsexterne Faktoren ein, die nicht mehr »rational rekonstruierbar« sind, sondern sozialgeschichtlich untersucht werden müssen.

Dem Versuch, die (angebliche) Form naturwissenschaftlichen Wissens zum Maßstab von wissenschaftlichem Wissen überhaupt zu machen und insbesondere die Unwissenschaftlichkeit einer kritischen Theorie der Gesellschaft nachzuweisen, ist durch die hier nur angedeuteten Ergebnisse der wissenschaftshistorischen Forschung der Boden entzogen: die von den »kritischen Rationalisten « gepflegte Vorstellung von Wissenschaft existiert auch in den Naturwissenschaften nicht, es handelt sich bei dieșer Vorstellung um ein allerdings einflußreiches - Hirngespinst. Umgekehrt verweisen die »externen « Faktoren, die für die Durchsetzung eines neuen Paradigmas notwendig sind, darauf, daß sich die naturwissenschaftlichen Formen des Wissens nicht aus sich selbst heraus begreifen lassen, sondern auf eine sozialwissenschaftliche Aufklärung angewiesen sind.

\section{Zur Sozialgeschichte der naturwissenschaftichen Form des Wissens}

Bereits aus den 30er Jahren datieren Versuche, die Naturwissenschaften sozialwissenschaftlich zu untersuchen. So stellten insbesondere frühe marxistische Ansätze einen Zusammenhang zwischen der Herausbildung des Kapitalismus und den neuzeitlichen Naturwissenschaften her. In seiner für das Frankfurter »Institut für Sozialforschung « angefertigten umfangreichen Studie »Der Übergang vom feudalen zum bürgerlichen Weltbild« geht Franz Borkenau davon aus, daß die grundlegenden Denkformen der Naturwissenschaft (wie der Begriff des Naturgesetzes, der Kausalität etc.) keine ewigen, überhistorischen, sondern historische Formen sind, und daß das naturwissenschaftliche Weltbild daher nicht einfach ein »natürliches « Abbild der Außenwelt ist, sondern in seiner begrifflichen Grundstruktur historisch produziert ist und daher auch historisch erklärt werden muß.

Das »mechanistische Weltbild«, das sich nach Borkenaus Auffassung im 17. Jahrhun- 
dert mit der Philosophie von Descartes durchgesetzt hat, soll nach dem Vorbild der Manufaktur konstruiert sein: »Ganz grob ausgedrückt, läßt sich das Bestreben, das ganze Naturgeschehen aus mechanischen Prozessen zu erklären, als die Bemühung definieren, alles Naturgeschehen nach Analogie der Vorgänge in einer Manufaktur aufzufassen.«(Borkenau 1934, S. 5) Die Durchsetzung dieses mechanistischen Weltbildes lasse sich aber nicht einfach auf die Entwicklung der Produktivkräfte zurückführen, sondern auf die gesellschaftlichen Umwälzungen dieser Zeit (ebd. S. 13 f.). Die sozialen Einflüsse versuchte Borkenau, besonders an der Entwicklung des Begriffs des Naturgesetzes seit der Hochscholastik zu verfolgen (vergl. auch Borkenau 1932). Er kam dabei zu dem Ergebnis, daß die an quantitativen Bestimmungen orientierte Naturwissenschaft erst mit der Entwicklung der Manufakturen möglich geworden sei.

In einer ausführlichen Auseinandersetzung mit Borkenaus Buch zeigte Henryk Grossmann (1935) eine Reihe von sowohl historischen als auch wissenschaftsgeschichtlichen Unzulänglichkeiten auf. Vor allem datierte er die Entstehung der modernen Mechanik wesentlich früher, nämlich mit Leonardo da Vinci und widersprach Borkenaus Grundthese, das mechanistische Weltbild sei nach dem Vorbild der Manufaktur konstruiert. Im Gegensatz dazu versuchte Grossmann nachzuweisen, daß die Mechanik und das mechanistische Weltbild auf der Grundlage der Entwicklung der Maschinen entstanden ist.

Während Borkenau und Grossmann wenigstens noch in Ansätzen versuchten, die spezifischen Formen des neuzeitlichen naturwissenschaftlichen Wissens zu erfassen, begnügten sich die übrigen Versuche einer sozialgeschichtlichen Erforschung der Naturwissenschaften mehr oder weniger mit einer »Einflußforschung «: sie zielten in erster Linie auf die gesellschaftlichen Faktoren ab, die die Entwicklung der Naturwissenschaften begünstigt hatten. So hatte Boris Hessen bereits 1931 anhand der Newtonschen Mechanik zu zeigen versucht, daß sich die neuzeitliche Naturwissenschaft aufgrund der Bedürfnisse der materiellen Produktion und des Verkehrs entwickelt hatte. ${ }^{6}$ In eine ähnliche Richtung wiesen auch die Untersuchungen von Bernal (1954). In diesen Konzeptionen wird der Nutzen, den die Naturwissenschaften für die Probleme des sich entwicklenden Kapitalismus bringen, bereits als Erklärung für deren Entstehung betrachtet.

Auch von nicht-marxistischen Wissenschaftlern wurde die Entstehung der neuzeitlichen Naturwissenschaften soziologisch untersucht. Dabei zielen die frühen Arbeiten von Merton auf eine ähnliche »Einflußforschung « wie sie auch bei Hessen oder Bernal vorliegt. Merton untersuchte am England des 17. Jahrhunderts die sozialen Bedingungen der Entstehung und Institutionalisierung der Naturwissenschaft. Dabei betrachtete er nicht nur den Einfluß ökonomischer und militärischer Interessen auf die Wahl der Forschungsprobleme, sondern auch die Bedeutung kultureller Wertvorstellungen, wie etwa die Rolle des Puritanismus. Gerade in der Aufwertung von allen Arten der praktischen Tätigkeit durch den Puritanismus sah Merton (1938) die Voraussetzung dafür, daß sich das Baconsche Programm einer experimentellen Naturwissenschaft überhaupt durchsetzen konnte. Den ökonomischen und kulturellen Fak- 
toren schrieb Merton zwar einen Einfluß auf das Schicksal von Theorien zu, die Frage, ob sie auch Einflüsse auf die »interne« Rationalität dieser Theorien haben könnten, klammerte er jedoch aus. Insofern ist seine spätere Wende zu rein wissenschaftssoziologischen Fragestellungen, wie etwa der nach den institutionellen Bedingungen des langfristigen Wachstums wissenschaftlichen Wissens unter völliger Ausblendung der kognitiven Struktur dieses Wissens, keineswegs überraschend.

Edgar Zilsel, der dem Wiener Kreis nahestand, versuchte die spezifischen Merkmale der modemen Naturwissenschaften, daß Gesetze der Natur gesucht werden, daß dies auf experimentellem Wege geschieht und daß die Forschungsergebnisse als Fortschritt gelten, auf verschiedene soziale Träger zurückzuführen: die scholastischen Gelehrten, die Handwerker-Ingenieure und die Humanisten. Erst ihr Zusammenwachsen in der entstehenden Stadtkultur brachte dann die neuzeitliche Naturwissenschaft hervor. Zilsel erklärt damit aber nicht die Form naturwissenschaftlichen Wissens, er sucht nach soziologischen Gesetzen, die das Auftreten dieser Wissensform determinieren oder zumindest wahrscheinlich machen.

In diesen soziologischen Untersuchungen der Entstehung der modernen Naturwissenschaften bleibt die Frage nach der Konstitution der spezifischen Form naturwissenschaftlichen Wissens weitgehend ausgeblendet. Es scheint, als habe es lediglich eines äußeren Anstoßes bedurft, um diese Form zu entwickeln. Sie selbst erscheint aber die dem Gegenstand, der Natur, angemessene und daher überzeitliche Form zu sein.?

Die Konsequenz einer derartigen Auffassung ist die systematische Trennung zwischen Wissenschaftstheorie, die die kognitiven Strukturen wissenschaftlichen Wissens erforscht und Wissenschaftssoziologie, die unter Abstraktion von den kognitiven Strukturen der Wissenschaft die sozialen Prozesse, die die Wissenschaftsentwicklung beeinflussen, untersucht. Die Wissenschaftsgeschichte zerfällt dann in eine »interne « und eine »externe « Geschichte: die interne Geschichte ist die Geschichte der Akkumulation und Veränderung des wissenschaftlichen Wissens, die externe Geschichte stellt die beschleunigende oder hemmende Wirkung des sozialen Umfeldes dar.

Wie aber im vorangegangenen Abschnitt deutlich geworden sein sollte, läßt sich eine solche Trennung zwischen Wissenschaftstheorie und Wissenschaftssoziologie, zwischen externer und interner Geschichte nicht ohne weiteres aufrecht erhalten.

Die soziale Konstituierung der Form naturwissenschaftlicher Erkenntnis wurde von Sohn-Rethel zum Gegenstand verschiedener Arbeiten gemacht (Sohn-Rethel 1961, 1970, 1976). Sohn-Rethel betont den nicht-empirischen Gehalt der abstrakt mathematischen Denkform, die der Formulierung naturwissenschaftlicher Gesetze zugrunde liegt. Diese Denkform will er gesellschaftlich erklären, ihren Ursprung erblickt er in der Realabstraktion, die beim Warentausch vonstatten geht. Das Vermittlungsglied zwischen Warenform und Denkform sieht er im Geld. Das gemünzte Geld faßt er als die Materialisierung derTauschabstraktion auf, in ihm wird diese Abstraktion sinnlich wahrnehmbar und liefert der Philosophie das Muster für ihre Abstraktionsbildungen (Sohn-Rethel 1976, S. 41).

Zwar gelingt es Sohn-Rethel, eine Reihe von Analogien zwischen der Realabstrak- 
tion der »Tauschhandlung « und den mathematisch-quantitativen Begriffsbildungen aufzuzeigen. So weist er daraufhin, daß, wenn beim Tausch von den konkreten Gebrauchswerten der Waren abstrahiert und diese auf abstrakte Wertquantitäten reduziert werden, dies die Form des mathematischen Denkens sei, das mit von jeder Qualität abgelösten Quantitäten rechne (Sohn-Rethel 1970, S. 74 ff.). Daß die begrifflichen Abstraktionen aber tatsächlich durch die Tauschabstraktionen erzeugt werden, kann er nicht nachweisen. Als ein solcher Nachweis genügt ihm schon die Strukturidentität zwischen Tauschabstraktion und Denkabstraktion. ${ }^{8}$ Diese Strukturidentität kann er aber oft nur durch starke Abstraktionen, die nicht immer gerechtfertigt erscheinen, aufzeigen. Die soziale Genese der naturwissenschaftlichen Denkform wird auf diese Weise nicht erklärt.

$\mathrm{Da} 3$ der von Sohn-Rethel eingeschlagene Weg, direkt von der Tauschabstraktion zu mathematischen und naturwissenschaftlichen Begriffsformen zu gelangen, kaum gangbar ist, wird auch im Ahistorismus seines Ansatzes deutlich. Mit der Einführung des gemünzten Geldes im siebten vorchristlichen Jahrhundert ist für Sohn-Rethel die Grundlage der abstrakten Denkformen gegeben. Aristoteles und Galilei werden gleichermaßen zu Naturwissenschaftlern, deren unterschiedliche Konzeptionen nicht mehr einsichtig zu machen sind. So versucht Sohn-Rethel zwar aufzuzeigen, daß das Konzept der Inertialbewegung, das der Galilei-Newtonschen Physik als nicht-empirisches Prinzip zugrundeliegt, strukturidentisch mit der abstrakten Tauschbewegung ist. Warum es aber nicht schon von Aristoteles entdeckt wurde, ist in seinem Ansatz nicht mehr nachzuvollziehen. ${ }^{9}$

Besonders von kritischen Naturwissenschaftlem wurden Sohn-Rethels Thesen in den 70er Jahren intensiv diskutiert. Dabei wurde vor allem kritisch vermerkt, daß SohnRethel das Experiment als Ort der naturwissenschaftlichen Erkenntnisgewinnung nicht berücksichtigt (z.B. Hieber 1977, Dudek 1979). Damit schien zugleich eine Möglichkeit gegeben, Sohn-Rethels Beschränkung auf die Zirkulation als einziger Sphäre, die die Denkformen generiert, zu überwinden und Wissenschaft als »allgemeine Arbeit« aufzufassen. Allerdings kamen auch diese Ansätze nicht über die Bildung allgemeiner Analogien zwischen Warenproduktion und dem naturwissenschaftlichen Experiment hinaus. ${ }^{10}$

Soll sich die sozialgeschichtliche Rekonstruktion naturwissenschaftlicher Denkformen nicht auf eine bloße Analogiebildung beschränken, so ist eine differenziertere Untersuchung notwendig, als man sie bei Sohn-Rethel findet. Weder lassen sich die gesellschaftlichen Verhältnisse ganz allgemein auf den Warentausch noch die naturwissenschaftlichen Denkformen auf abstrakt quantifizierende reduzieren. Vor allem muß aber versucht werden, Ansatzpunkte für die Genese der je spezifischen Denkformen aus ihrem besonderen sozialen Kontext zu gewinnen. Die Ergebnisse einer solchen Untersuchung umfassen dann zwar nicht »die« Naturwissenschaft, sondern lediglich bestimmte Begriffsbildungen, die in einzelnen Phasen oder Umbrüchen eine Rolle gespielt haben, diese Ergebnisse sind aber aussagekräftiger als bloße Analogiebildungen. Bereits vor einigen Jahren sind zwei, bisher kaum rezipierte Arbeiten entstanden, die in eine solche Richtung weisen. Es handelt sich dabei und die »Geschich- 
te der Impetustheorie« von Michael Wolff und »Atom und Individuum im Zeitalter Newtons « von Gideon Freudenthal.

Wolff kritisiert die weithin akzeptierte Trennung von »externer« und »interner « Geschichte der Wissenschaften: ob ein Ereignis zur internen oder zur externen Geschichte gehört, hängt vom zugrundeliegende Begriff der jeweiligen Wissenschaft $\mathrm{ab}$, und dieser ändert sich mit dem jeweiligen Forschungsstand. Er selbst versucht, quer zu dieser Trennung eine »genetische Historiographie« eines bestimmten Abschnitts der Herausbildung der neuzeitlichen Naturwissenschaft zu liefern. Ausgangspunkt seiner Überlegungen ist, daß die Übergänge zwischen den großen physikalischen Paradigmen (der aristotelischen Physik der Antike, der Impetusphysik des Mittelalters und der Galilei-Newtonschen Physik der Neuzeit) nicht empirisch begründet werden können. Vielmehr stellen die Grundprinzipien dieser Paradigmen nicht-empirische Begriffsbildungen dar, die eine bestimmte Empirie erst strukturieren. Diese Prinzipien (Berührungskausalität, Übertragungskausalität, Trägheitsmechanik) erscheinen in der Blütezeit des Paradigmas aber jeweils als evidente Wahrheiten. Wolff untersucht nun am Beispiel der Impetustheorie das Problem, wie diese Evidenzen produziert werden. Die Lösung findet er in bereits begrifflich strukturierten, aber noch vortheoretischen, überindividuellen Motiven, die begriffsbildend in die Wissenschaft eingehen. Diese überindividuellen Motive bezeichnet Wolff als den »sozialen Kontext« einer wissenschaftlichen Entwicklung (Wolff 1978, S. 59).

Wie Wolff sein Programm realisiert, soll hier anhand seiner Deutung der Entstehung der Impetustheorie skizziert werden. Die Impetustheorie, die im 6. Jahrhundert in Johannes Philoponos ihren ersten bedeutenden Repräsentanten findet, ersetzt das der aristotelischen Bewegungslehre zugrundliegende Prinzip der »Berührungskausalität « durch das der »Übertragungskausalität«: danach entsteht Bewegung durch die Übertragung einer Kraft von einem Beweger auf das Bewegte, während in der aristotelischen Physik die Bewegung durch den dauernden Kontakt mit der Bewegungsursache hervorgebracht wurde. Für beide Prinzipien gilt, daß sie nicht empirisch begründet werden können. lhre Ablösung läßt sich daher auch nicht mit dem Verweis auf irgendwelche Beobachtungen rechtfertigen. Wolff sucht nun nach dem sozialen Kontext der Entstehung und Durchsetzung des Prinzips der Übertragungskausalität. Das Prinzip einer übertragenen Kraft findet Wolff bei Philoponos auch in anderen literarischen Kontexten wieder, in theologischen und vor allem in einem ökonomischen: der Kaufakt eines Produkts wird als Bezahlung der Kraft, die durch die Produktion auf das Produkt übertragen wurde, gedeutet. Daraus ergibt sich die Forderung nach einem »gerechten Preis«. Hier sieht Wolff eine Verbindung zum Emanzipationskampf der Handwerker in der zerfallenden Sklavenhaltergesellschaft der Spätantike. Die Forderung der Handwerker nach einem $»$ gerechten Preise « für ihre Produkte wird durch die Vorstellung legitimiert, der Produzent überträgt eine bestimmte Kraft auf das Produkt, die bezahlt werden muß. Das Prinzip der Übertragungskausalität gewinnt seine $»$ Evidenz« damit aus einer bestimmten spätantiken Emanzipationsideologie. Wolff behauptet nun aber nicht, daß die naturphilosophische Begriffsbildung ihre Ursache in der ökonomischen habe, sondern daß die Vorstellung der Kraftüber- 
tragung ein aus bestimmten Interessen zu erklärendes vortheoretisches Motiv ist, ein Denktypus, der in den verschiedenen Bereichen des Wissens die Begriffsbildung strukturiert.

Einem ähnlichen Erklärungsansatz wie Wolff folgt auch Freudenthal. Er versucht nachzuweisen, daß bestimmte Begriffsbildungen bei Newton auf philosophischen Vorbegriffen, die als evident angesehen werden, beruhen und daß diese Vorbegriffe ihrerseits verallgemeinerter Ausdruck von bestimmten sozialen Erfahrungen sind. Freudenthal führt dieses Programm anhand von Newtons Konstruktion des »absoluten Raumes « durch. Er zeigt, daß Newtons Beweise für die Existenz des absoluten Raums nicht nur auf der Voraussetzung beruht, daß Phänomene aus den Eigenschaften von Partikeln folgen, sondern auch darauf, daß jedes Partikel seine »essentiellen Eigenschaften « auch dann besitzt, wenn es allein im leeren Raum existieren würde. Letztere Auffassung erscheint Newton anscheinend als so selbstverständlich, daß er sie nicht einmal erwähnt. Newton geht also von einem bestimmten als evident angenommenen Vorbegriff über das Verhältnis von System und Element aus: den Elementen eines Systems kommen ihre wesentlichen Eigenschaften unabhängig von dem System zu. Newtons Raumtheorie wurde aber nicht nur aufgrund dieses Vorbegriffs konstruiert, Freudenthal kann auch plausibel machen, daß sie sich gerade aufgrund dieses »evidenten« Vorbegriffs durchsetzte.

Die Vorstellung, daß die Elemente eines Systems ihre Eigenschaften unabhängig von diesem System besitzen, findet sich auch in den Sozialtheorien des 17. und 18. Jahrhunderts wieder. Für den Bereich der Sozialtheorien sieht Freudenthal die Evidenz dieses Vorbegriffs in der sich herausbildenden bürgerlichen Gesellschaft begründet: sie führt zu der Vorstellung, die Gesellschaft sei aus Individuen zusammengesetzt, die ihre Eigenschaften unabhängig von der Gesellschaft bereits in sich tragen würden. Freudenthal behauptet allerdings nicht, daß Newton den Gegenstand seiner Naturtheorie einer sozialphilosophischen Denkform entsprechend modelliert habe, 'sondern daß in beiden Bereichen derselbe philosophische Grundsatz über das Verhältnis von System und Element angewendet wurde und daß die Evidenz dieses Grundsatzes aus den sozialen Verhältnissen folgte.

Jeder Versuch, die soziale Genesis der naturwissenschaflichen Denkformen zu erklären, sieht sich mit dem Wahrheitsanspruch der Naturwissenschaften konfrontiert. Bedeutet ein solcher Versuch den Verzicht auf diesen Wahrheitsanspruch und damit auf wissenschaftliche Rationalität? Wird Naturwissenschaft zu einer beliebigen Auffassung von Natur, die sich nicht prinzipiell von irgendwelchen Mythen unterscheiden läßt? Eine solche Auffassung scheint dem Vorwurf des Irrationalismus oder Relativismus zugrunde zu liegen, der sozialgeschichtlichen Erklärungsversuchen naturwissenschaftlicher Denkformen häufig gemacht wird.

Allerdings verdankt sich dieser Wahrheitsanspruch der Hypostasierung einer fiktiven erkenntnistheoretischen Situation: das Individuum steht als isoliertes Erkenntnissubjekt dem zu erkennenden Objekt ohne jede Vermittlung gegenüber und will dieses von ihm isolierte Objekt erkennen. Die Erkenntnis kann dann dem Objekt entweder angemessen oder unangemessen sein, sie kann wahr oder falsch sein. Daß sich Subjekt und 
Objekt nicht unvermittelt gegenüberstehen, wurde bereits vom Deutschen Idealismus erkannt. Allerdings beschränkte sich die Vermittlung dort auf das Denken. Demgegenüber machte Marx die gesellschaftliche Praxis als entscheidende Vermittlungsinstanz geltend. ${ }^{11}$ In ihr sind Subjekt und Objekt immer schon als Momente einer vermittelten Einheit vorhanden. Ihre Isolierung ist ein Produkt der Abstraktion. Weder sind die Subjekte der Erkenntnis unbeschriebene Blätter, noch sind die Objekte der Erkenntnis an sich gegebene Dinge, die bloß angeschaut werden müßten. Was als auch wie etwas zum Gegenstand der Erkenntnistätigkeit wird, ist gesellschaftlich vermittelt. Von Wahrheit kann daher auch nur innerhalb dieser Vermittlung gesprochen werden. Für die Naturwissenschaft bedeutet dies, daß sowohl die Art und Weise wie Natur zum Gegenstand von Wissenschaft wird, als auch das, was als Wissenschaft gilt, gesellschaftlich konstituiert ist. »Natur « ist nicht an sich gegeben, sie wird immer schon mittels nicht-empirischer Vorbegriffe (wie etwas dem des Naturgesetzes oder dem der Trägheitsbewegung), die die Empirie erst strukturieren, aufgefaßt. Und die Evidenz solcher Vorbegriffe findet ihre Erklärung im sozialen Kontext. Ebenso sind die Geltungskriterien vom jeweiligen Wissenschaftsbegriff abhängig, und dieser ist ein historisches Resultat. Wird dagegen als Beweis für die überhistorische Wahrheit der Naturwissenschaft auf die Erfolge der auf ihr aufbauenden Technik verwiesen, so wird die technische Beherrschbarkeit der Natur zum evidenten Kriterium der Wahrheit gemacht. Daß auch diese Evidenz historisch produziert ist, wird klar, wenn man bedenkt, daß technische Beherrschbarkeit der Natur einem antiken Philosophen als Wahrheitskriterium kaum eingeleuchtet hätte.

Die Naturwissenschaft ist also keineswegs die »Naturform « des Wissens über die Natur; sie ist ebensowenig wie andere geistige Formen unabhängig vom sozialen Lebensprozeß, was von all denen übersehen wird, die wie Popper, den Versuch unternehmen, eine zeitlose Methodologie aus ihr zu abstrahieren. Die neuzeitliche Naturwissenschaft ist Naturwissenschaft der bürgerlichen Gesellschaft. Das bedeutet nun aber nicht, daß man einer »bürgerlichen «Physik eine »proletarische« gegenüberstellen könnte. Damit ist vielmehr ausgedrückt, daß den naturwissenschaftlichen Begriffsbildungen, ähnlich wie denen der klassischen politischen Ökonomie, »objekcive Gedankenformen « (Marx) zugrunde liegen, die in der bürgerlichen Gesellschaft ihre Grundlage haben.

\section{Anmerkungen}

1 Das heißt, Kopernikus wurde weniger von den Epizykeln, die er auch selbst verwandte, als von den bei Ptolemäus benutzten Äquanten gestört und er betrachtete es als seine größte Leistung, die Äquanten eliminiert zu haben (vergl.: Dijsterhuis 1950, S. 321).

2 Fast dieselben Argumente führt auch Lakatos (1976, S. 199 ff.) an, um zu zeigen, daß das Kopernikanische Programm gegenüber dem Ptolemäischen einen objektiven wissenschaftlichen Fortschritt darstellte.

3 Daß das gesellschaftliche Umfeld nicht nur für die Entstehung der neuzeitlichen Naturwissenschaft eine Rolle spielte, sondern beispielsweise auch für die Entwicklung der Quantentheorie in der Weimarer Republik, machte Forman (1968) deutlich. 
4 Während Kuhn und Lakatos auf einer eher globalen Ebene argumentieren, untersuchte Knorr-Cetina (1984) den Mikrokosmos des Forschungsalltags. Auch ihre auf teilnehmender Beobachtung beruhende anthropologische Studie über die Sitten und Gebräuche bei der »Fabrikation von Erkenntnis« in einem biochemischen Labor in Berkeley zeigt, daß die Idealvorstellungen der Wissenschaftstheorie nicht viel mit der Praxis naturwissenschaftlicher Forschung zu tun haben.

5 Eine solche Projektion existiert auch über die Entstehung der speziellen Relativitäts theorie: angeblich sei die klassische Äthertheorie durch den Versuch von Michelson und Morley zu Fall gebracht worden, woraufhin Einstein seine spezielle Relativitätstheorie entwickelt habe. Dieser Interpretation steht aber entgegen, daß die Diskussion über die Bedeutung des Michelson-Morley Versuchs einerseits noch gar nicht abgeschlossen war, als Einstein 1905 seinen berühmten Artikel publizierte und daß andererseits Einstein mit keinem Wort auf diesen Versuch oder iiberhaupt nur auf eine $\gg$ Falsifizierung « der Äthertheorie eingeht. Ihm ging es vielmehr um die mangelnde Symmetrie bei der Lösung eines bereits seit langem bekannten (und mit dem klassischen Apparat gelösten) Problems (vergl. dazu auch Holton 1981).

6 Lefèvre (1978) macht im Gegensatz dazu plausibel, daß es vor dem 18. Jahrhundert kein von der materiellen Produktion ausgehendes Bedürfnis nach wissenschaftlichen Lösungen hat geben können, da die Produktion auf handwerklicher Grundlage beruhte, die eine Nutzung allgemeiner wissenschaftlicher Erkenntnisse gerade ausschloß.

7 Hier zeigt sich eine Parallele zu der Art und Weise, wie die Geschichtlichkeit des Kapitalismus in der klassischen und neoklassischen Ökonomie aufgefaßt wird. Zwar wird gesehen, daß der Kapitalismus historisch entstanden ist, er gilt dann aber ganz unhistorisch als die »natürliche« Form der Produktion.

8 Explizit erklärt er: »Ja, im Grunde sollte der Nachweis solcher Identität allein schon genügen, um die Genesis der Verstandeskategorien aus der Realabstraktion zur theoretischen Gewißheit zu machen, gleichgültig, wie dieselbe sich immer plausibel machen oder geschichtlich erklären läßt.« (SohnRethel 1976, S. 57)

9. Zur Begründung führt Sohn-Rethel an: »Die Ursache ist offenkundig die Bindung des aristotelischen Denkens an die Arbeit als Sklavenarbeit, während der mittelalterliche Produzent als Handwerker oder Bauer, ob frei oder unfrei, sich im Besitz seiner Arbeitsmittel befindet, so daß für ihn die Bewegungsursache der Dinge nicht mehr an die Leibestätigkeit seiner Person gekettet sein braucht.« (SohnRethel 1976, S. 53) Abgesehen vom bloß pauschalen Charakter dieses Arguments besteht auch keine zeitliche Koinzidenz - zur Zeit Galileis gehörten die Sklavenhaltergesellschaften auch schon einige Jahrhunderte der Vergangenheit an.

10 So sieht Dudek im Experiment einerseits »abstrakt allgemeine Arbeit « am Werk, andrerseits stellt er eine »Koinzidenz zwischen der Form des Messens und der Form des Tauschens« (Dudek 1979, S. 152) fest - nämlich insofern als beiden »Formen « eine Äquivalenzrelation unterliegt.

11 Bereits in der ersten These über Feuerbach warf Marx dem »bisherigen« Materialismus vor, nicht gesehen zu haben, daß alle objektive Wirklichkeit subjektiv vermittelt ist: »Der Hauptmangel alles bisherigen Materialismus (den Feuerbachschen mit eingerechnet) ist, daß der Gegenstand, die Wirklichkeit, Sinnlichkeit nur unter der Form des Objekts oder der Anschauung gefaßt wird; nicht aber als sinnlich menschliche Tätigkeit, Praxis; nicht subjektiv. Daher die tätige Seite abstrakt im Gegensatz zu dem Materialismus von dem Idealismus - der natürlich die wirkliche, sinnliche Tätigkeit als solche nicht kenne - entwickelt.« (MEW 3, S. 5)

\section{Literatur}

Adorno, Theodor W. (1961): Zur Logik der Sozialwissenschaften, in: Adorno u.a. (1972), S. 125-144 Adorno, Theodor W. u.a. (1972): Der Positivismusstreit in der deutschen Soziologie, Darmstadt und Neuwied

Bernal, J.D. (1954): Science in History (deutsche Ausgabe), Hamburg 1970

Borkenau, Franz (1932): Zur Soziologie des mechanistischen Weltbildes, in: Zeitschrift für Sozialforschung, Jahrgang I, Heft 3, S. 311-355 
Borkenau, Franz (1934): Der Übergang vom feudalen zum bürgerlichen Weltbild. Studien zur Geschichte der Philosophie der Manufakturperiode, Paris

Butterfield, Herbert (1966): The Origins of Modern Science 1300-1800, New York

Carnap, Rudolf (1928): Der logische Aufbau der Welt, Berlin

Dijsterhuis, E.J. (1950): Die Mechanisierung des Weltbildes, Berlin 1983

Dudek, Peter (1979): Naturwissenschaftliche Denkformen und ökonomische Struktur, in: PROKLA 34, S. $127-157$

Feyerabend, Paul (1975): Wider den Methodenzwang, Neuausgabe Frankfurt 1986

Forman, Paul (1968): The Environment and Practice of Atomic Physics in Weimar Germany: A Study in the History of Science, University of California, Berkeley

FreudenthaI, Gideon (1982): Atom und Individuum im Zeitalter Newtons. Zur Genese der mechanistischen Natur- und Sozialphilosophie, Frankfurt

Grossmann, Henryk (1935): Die gesellschaftlichem Grundlagen der mechanistischen Philosophie und die Manufaktur, in: Zeitschrift für Sozialforschung, Jahrgang IV, Heft 2, S. 161-231

Habermas, Jürgen (1963): Analytische Wissenschaftstheorie und Dialektik, in Adorno u.a. (1972), S. 155192

Habermas, Jürgen (1965): Erkenntnis und Interesse, in: ders., Technik und Wissenschaft als Ideologie, Frankfurt 1974, S. 146-168

Hessen, Boris (1931): Die sozialen und ökonomischen Wurzeln von Newtons »Principia«, in: Peter Weingart (Hrsg.) Wissenschaftssoziologie II, Frankfurt 1974, S. 262-325

Hieber, Lutz (1977): Sohn-Rethels Bedeutung für die Selbstreflexion naturwissenschaftlicher Arbeit, in; PROKLA 29, S. 77-99

Holton, Gerald (1981): Einstein, Michelson und das experimentum crusis, in: ders., Thematische Analyse der Wissenschaft. Die Physik Einsteins und seiner Zeit, Frankfurt, S. 255-371

Knorr-Cetina, Karin (1984): Die Fabrikation von Erkenntnis. Zur Anthropologie der Naturwissenschaft, Frankfurt

Koyré, Alexandre (1939): Études Galiléene, Paris

Kuhn, Thomas S. (1957): Die kopernikanische Revolution, Braunschweig 1981

Kuhn, Thomas S. (1962): Die Struktur wissenschaftlicher Revolutionen, zweite revidierte Auflage, Frankfurt 1976

Lakatos, Imre (1976): Warum hat das Kopernikanische Forschungsprogramm das Ptolemäische überrundet? in: ders., Philosophische Schriften Bd. 1, Braunschweig 1982, S. 182-208

Lakatos, Imre (1968): Falsifikation und die Methodologie wissenschaftlicher Forschungsprogramme, in: ders., Philosophische Schriften Bd. 1, Braunschweig 1982, S. 7-107

Lakatos, Imre (1970): Die Geschichte der Wissenschaft und ihre rationalen Rekonstruktionen, in: ders., Philosophische Schriften Bd. 1, Braunschweig 1982, S. 108-148

Lefèvre, Wolfgang (1978): Naturtheorie und Produktionsweise. Probleme einer materialistischen Wissenschaftsgeschichtsschreibung - Eine Studie zur Genese der neuzeitlichen Naturwissenschaft, Darmstadt und Neuwied

Maier, Anneliese (1949): Die Vorläufer Galileis im 14. Jahrhundert, Rom 1966

Merton, Robert K. (1938): Science, Technology und Society in Seventeenth Century England, New York 1970

Popper, Karl (1935): Logik der Forschung, Tübingen 1984

Popper, Karl (1961): Die Logik der Sozialwissenschaften, in Adorno u.a. (1972), S. 103-124

Popper, Karl (1969): Conjectures and Refutations. The Growth of Scientific Knowledge, Third Edition (revised) London

Rickert, Heinrich (1902): Die Grenzen der naturwissenschaftlichen Begriffsbildung, Tübingen und Leipzig

Sohn-Rethel, Alfred (1961): Warenform und Denkform. Versuch über den gesellschaftlichen Ursprung des »reinen Verstandes«, in: ders., Warenform und Denkform, Frankfurt 1978, S. 103-133

Sohn-Rethel, Alfred (1970): Geistige und körperliche Arbeit. Zur Theorie der gesellschaftlichen Synthesis, 2. erweiterte Auflage, Frankfurt 1973

Sohn-Rethel, Alfred (1976: Das Geld, die bare Münze des Apriori, in: Paul Mattick, Alfred Sohn-Rethel, Hellmut G. Haasis, Beiträge zur Kritik des Geldes, Frankfurt, S. 35-117 
Weber, Max (1922): Wirtschaft und Gesellschaft, Tübingen 1972

Windelband, Wilhelm (1896): Geschichte der Naturwissenschaft, Freiburg

Wittgenstein, Ludwig (1921): Tractatus logico-philosophicus, Frankfurt 1963

Wolff, Michael (1978): Geschichte der Impetustheorie. Untersuchungen zum Ursprung der klassischen Mechanik, Frankfurt

Zilsel, Edgar (1985): Die sozialen Ursprünge der neuzeitlichen Wissenschaft. Hrsg. Wolfgang Krohn, Frankfurt (enthält Aufsätze aus den Jahren 1940-45) 